Ocean Engineering

January 2018, Volume 148 Pages 115-124

http://dx.doi.org/10.1016/i.oceaneng.2017.11.002

http://archimer.ifremer.fr/doc/00411/52205/

(c) 2017 Elsevier Ltd. All rights reserved.

\title{
Power estimates of an undulating membrane tidal energy converter
}

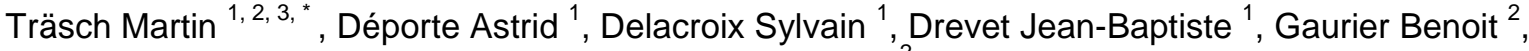 \\ Germain Gregory ${ }^{2}$
}

${ }^{1}$ Eel Energy, 133 bis rue de l'université, 75007 Paris, France

2 Ifremer, Marine Structure Laboratory, 150 Quai Gambetta, 62200 Boulogne sur Mer, France

${ }^{3}$ Ademe, 20 avenue du Gresille, 49004 Angers, France

*Corresponding author : Martin Träsch, email address : $\underline{\text { mtrasch@eel-energy.fr }}$

\begin{abstract}
:
An experimental study of an undulating membrane based on the Eel Energy device is presented in this paper. This system uses interaction between a semi-rigid plate and an axial flow to generate undulation. At full scale, the deformation of the structure is then converted into electrical power using linear electromagnetic generators. In order to simulate the power take-off on a 1/20th scale prototype, hydraulic dampers are located all over the length of the device. The dampers have non-linear behaviour. A representative damping parameter have been introduced to study their impact.

Results are presented in function of fluid velocity and damping adjustment. Undulation mode, frequency and forces on the structure are described. Results show that fluid velocity has a destabilizing effect on the membrane, increasing undulation frequency and lowering its amplitude, while damping has the opposite effect. Furthermore, two methods used to evaluate the power conversion are described and evaluated from trajectory and forces data analysis. Power estimation shows an evolution proportional to cubic current speed at low flow speed, as for classical tidal turbines. Power also seems to scale with the square of the product between undulation frequency and amplitude.
\end{abstract}

\section{Highlights}

- An experimental model of undulating membrane tidal energy converter at $1 / 20$ th scale is presented. - Power Take-Off simulation using hydraulic dampers is proposed and described. - Two methods of power conversion estimation have been described and evaluated. Membrane motion and forces are measured in function of current speed and damping adjustment Power conversion scales with the square product of undulation main frequency and motion amplitude. 


\section{Introduction}

Harvesting ocean's energy has been an idea seriously considered since the oil crisis in the 1970's. Before that, it was mentioned by french writer Jules Verne in the $19^{\text {th }}$ [Verne (1869)], whereas the energy of tides and rivers is being used since Antiquity [P.-L. Viollet (2005)]. Marine renewable energy is a relatively new field, and tidal converters are even more recent, although tidal currents present numerous advantages as being renewable, clean, predictable and available in high quantity. The sector is now in a strong growth dynamic and a lot of concepts are being developed, as reviewed by [Day (2015)]. Most of these concepts are inspired by turbines, whereas some are based on oscillating foils [Kinsey et al. (2011)], [Xiao and Zhu (2014)]. [Taylor (2001)] first introduced the concept of a flexible piezoelectric membrane placed in the wake of a bluff body. More recently, [Doare and Michelin (2011)] developed advanced model for small production piezoelectric membrane converters.

The aim of the undulating membrane tidal energy converter studied here is to use membrane deformation to activate electromagnetic converters (Figure 1). Therefore, it uses a strain wave generated by interactions between a semi-rigid structure and an axial flow. The membrane characteristics are chosen to be rigid enough to transmit the deformation force to converters and flexible enough to be deformed by the flow [Déporte (2015)]. It differs from the undulating devices cited earlier by its scale, about 15 meters, which is comparable with other classical tidal turbines. On the lateral direction, rigid profiles maintain the motion in two dimensions and avoid torsion modes which should be destructive.

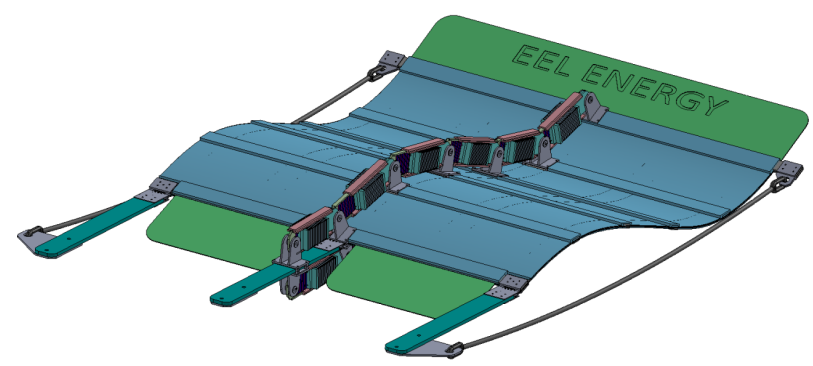

Figure 1: CAD drawing of an undulating tidal energy converter with electromechanical converters on the two sides along the center line and two compression cables.

The structure is pre-stressed by cables binding both extremities, buckled 
on its first deformation mode, and presents to the flow a significant surface. This configuration enables to lower the critical flow speed from which undulations start [Träsch et al. (2017)]. Below this velocity, a differential of pressure is observed for each side of the membrane but it is not strong enough to move it. When the critical speed is exceeded, differential of pressure increases and the membrane curvature reverses. The position becomes unstable and the strain wave is propagated from the leading edge to the trailing edge on second deformation mode. Cables have a major impact because they confine the motion and homogenize the deformation all over the length, enabling a better undulation control.

The undulations set in motion linear electromechanical converters distributed all over the length of the membrane. It uses linear converters instead of piezoelectric materials in order to produce high power [Jbaily and Yeung (2014)]. They are composed of several independent modules. The power dissipation could be adapted with the fluid flow all over the length of the membrane to optimize the energy uptake.

In this article, a $1 / 20^{\text {th }}$ scale prototype is tested in a flume tank. The experimental model is first described. Then, the membrane dynamic is assessed and power estimation is done on an operating point. The membrane's behaviour and performance are presented according to current speed and Power Take Off Level (PTO).

a.
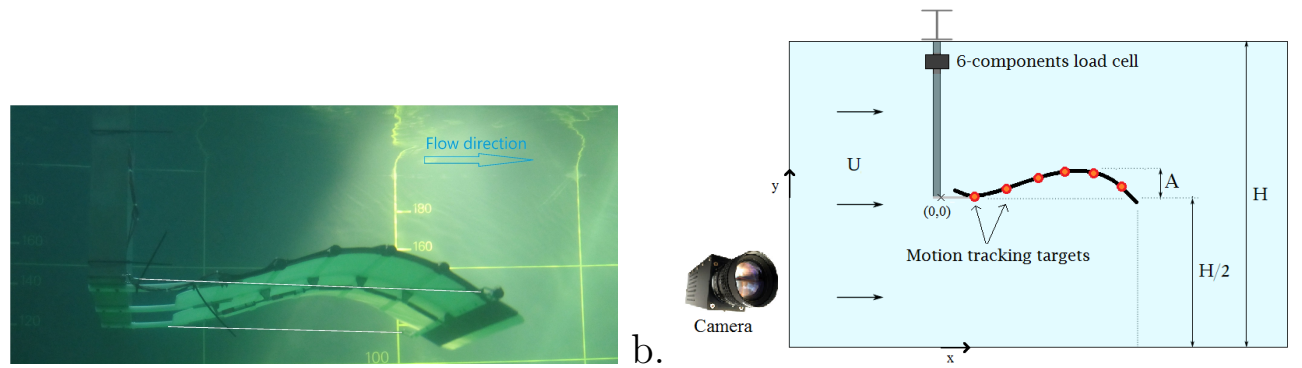

Figure 2: (a) Picture and (b) drawing of the 1:20 $0^{\text {th }}$ scale prototype and its instrumentation. 


\section{Material and method}

The experimental model is presented in this section. Small scale prototype dimensions and materials are first described, with a special focus on PTO modelisation. The converters' adjustment and behaviour are explained. Then, two processing methods are presented and compared. The flume tank characteristics and instrumentation are also addressed.

The prototype used in the experiments is made of a $L \times b \times h=0.8 \times$ $0.8 \times 0.004 \mathrm{~m}^{3}$ POM-C polyacetal membrane of density $\rho=1600 \mathrm{~kg} / \mathrm{m}^{3}$ and Young modulus $E=4.2 \mathrm{GPa}$ [Déporte (2015)]. Rigid flaps at upstream and downstream extremities lengthen the membrane by $L_{\text {flap }}=0.15 \mathrm{~L}$ each and enhance its undulation motion. Six $0.025 \mathrm{~L}$-wide carbon-epoxy bars have been added in the transverse direction in order to increase the transverse stiffness and to ensure a two-dimensional motion. They also transmit forces to the converters, that are linked to it by $h_{f i x}=0.044 L$-high fixations. Each fixation is separated by $0.2 \mathrm{~L}$ on the membrane and linked to dampers through pivot points.

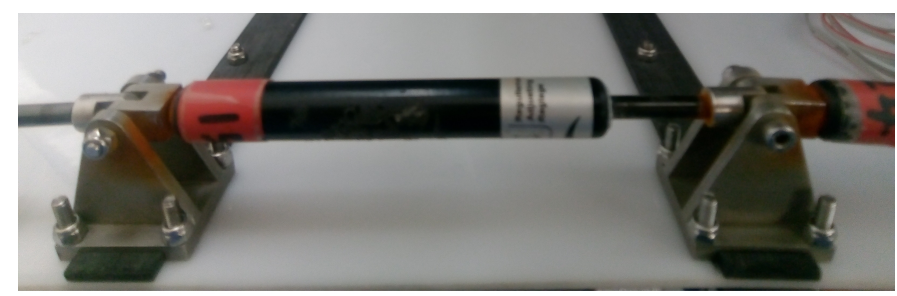

Figure 3: Picture of a hydraulic damper and its fixations on the membrane.

To keep the structure in the middle of the water column, the membrane is linked to a rigid vertical frame with three flexible arms of rigidity $k_{\text {arm }}=6250$ $\mathrm{N} / \mathrm{m}$. The structure is also stressed by cables linking both extremities that keep the membrane bended (Figure 2.a). The cables are $d=0.046 L$ shorter than the resting distance between their linking points.

At this scale $(1 / 20)$, it is easier to dissipate energy than to convert it into electricity. Therefore, for physical and mechanical constraints, electromechanical converters are modelled by hydraulic dampers, as was experimented for oscillating body devices in wave converter models [Day (2015)]. The dampers used in this study are the HB15/75/S/S/B from Slamproof Ltd. 
They are at the right dimensions, easy to supply and deliver an adjustable damping force according to their working speed.

The adjustment of damping force is made by pulling the piston rod until it stops and turning it. It screws the piston around the rod, moving it along forward (or backward), and covering (or discovering) the fluid path, leading to a higher (or lower) pressure drop. Dampers have been calibrated on a displacement table by operating them with a sinusoidal motion at fixed amplitude $( \pm 30 \mathrm{~mm})$ and frequency $(f=0.2 \mathrm{~Hz})$ during $60 \mathrm{~s}$. Figure 4 .a gives an example of period-averaged damping response from an hydraulic damper. A perfect converter model would show a force proportional to the damper speed. Here, however, there are non-linearities:

1. Hysteresis due to vapour bubbles that appear when piston volume rises because piston rod goes out and intern pressure is under vapour pressure $\left(\mathrm{P}_{\text {int }}<\mathrm{P}_{\text {vap }}\right)$,

2. A polynomial behaviour generated by point losses where cross section variations are sharp-edged $\left(\Delta \mathrm{P} \propto V^{2}\right)$,

3. Assymetric behaviour caused by differences in pressure drop and piston's working area.

a.
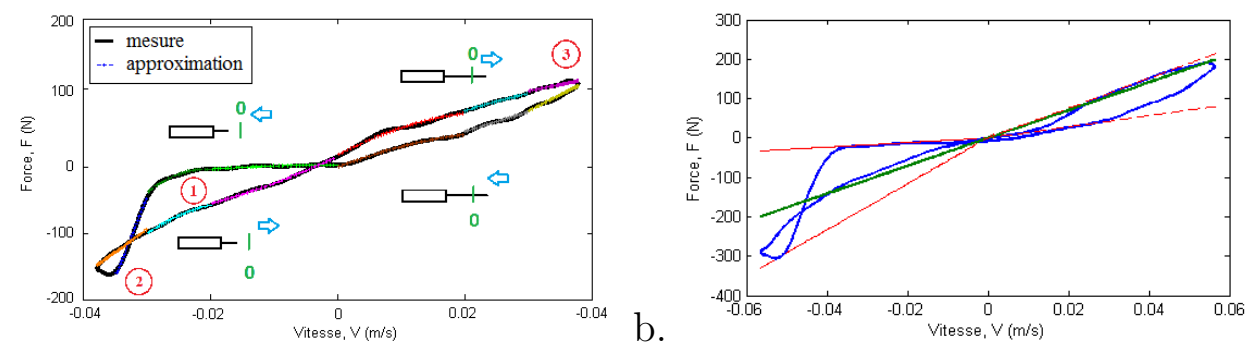

Figure 4: (a) Exemple of damping force opposed to periodic motion: $z(t)=0.03 \cos (0.4 \pi t)$. Damper symbols indicate damper's position and operating direction according to the position on the damping curve. Numbers in red $(1,2,3)$ refer to the list above the figure. (b) Exemple of linear approximation of damping force (black) and its uncertaincy (green).

Keeping in mind all these non-linearities, and in order to be able to give an order of magnitude of the damping rate for each damper, a representative damping parameter is introduced:

$$
c(k g / s)=\frac{\int_{t}^{t+1 / f} F(t) \cdot V(t) \mathrm{d} t}{\int_{t}^{t+1 / f} V(t)^{2} \mathrm{~d} t}
$$


This parameter is analogue to a damping coefficient but depends strongly on the displacement signal used for the damper characterisation. Yet, in the case of HB15/75 hydraulic dampers, and with sinusoidal displacement, it varies relatively little. However, the adjustment process results in high damping variation for low angle variation of the rod when the path is almost totaly covered by the piston. The standard deviation on the 36 dampers is then $\sigma_{3}=27.3 \%$, whereas the mean damping value is $\overline{C_{3}}=3058 \mathrm{~kg} / \mathrm{s}$.

$$
\begin{gathered}
C_{j}=\sum_{i=1}^{N_{\text {dampers }} c_{i}} \\
\overline{C_{j}}=\frac{\sum_{i=1}^{N_{\text {dampers }} c_{i}}}{N_{\text {dampers }}} \\
\sigma_{j}=\sqrt{\frac{\sum_{i=1}^{N_{\text {dampers }}\left(c_{i}-\overline{C_{j}}\right)^{2}}}{N_{\text {dampers }}{\overline{C_{j}}}^{2}}}
\end{gathered}
$$

The adjustment being complicated and uncertain, especially for high damping, the choice has been made to increase the number of dampers in order to increase the damping rate instead of changing the dampers' adjustment between each trial. Therefore, results presented hereafter correspond to trials with $0,1,2$ or 3 lines of dampers. They are respectively noted $C_{0}, C_{1}$, $C_{2}$ and $C_{3}$, as presented in Figure 5. The damping rates are defined as the sum of the damping parameters calculated by Equation 1 for each damper. This estimation is given along with mean value and standard deviation in Table 1 . Each line is made up of 12 dampers, add $\approx 36700 \mathrm{~kg} / \mathrm{s}$ total damping and $0.744 \mathrm{~kg}$ mass, being $9 \%$ of the total undamped membrane's mass.

\begin{tabular}{|c|c|c|c|c|}
\hline Configuration & $N_{\text {dampers }}$ & Damping rate & Mean value & Std deviation \\
\hline 1 line & 12 & $C_{1}=33450 \mathrm{~kg} / \mathrm{s}$ & $\overline{C_{1}}=2787 \mathrm{~kg} / \mathrm{s}$ & $\sigma_{1}=11.1 \%$ \\
\hline 2 lines & 24 & $C_{2}=76660 \mathrm{~kg} / \mathrm{s}$ & $\overline{C_{2}}=3194 \mathrm{~kg} / \mathrm{s}$ & $\sigma_{2}=30.6 \%$ \\
\hline 3 lines & 36 & $C_{3}=110100 \mathrm{~kg} / \mathrm{s}$ & $\overline{C_{3}}=3058 \mathrm{~kg} / \mathrm{s}$ & $\sigma_{3}=27.3 \%$ \\
\hline
\end{tabular}

Table 1: Damping characteristics of the tested configurations. 
$C_{0}$ is the configuration with no damper. Internal material damping of the membrane was estimated to be $C_{0}=1100 \mathrm{~kg} / \mathrm{s}$ by recording the frequency of its oscillations after a forced deflection. Kelvin-Voigt visco-elastic model was considered [Païdoussis (1998)]. Even if $C_{0}$ and $C_{1,2,3}$ have the same unit, they do not represent the same phenomenons and do not apply the same way on the membrane.
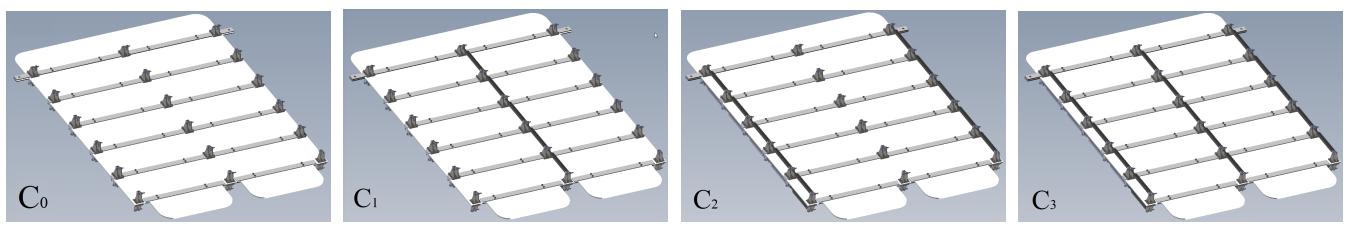

Figure 5: Dampers line repartition in tested configurations.

Two methods have been used to calculate the damping force and estimate the Power Take-Off [Déporte (2015)]. The linear method uses the coefficient $C(\mathrm{~kg} / \mathrm{s})$ defined in Equation 1. Trajectory data is used to estimate each damper's working speed. Instantaneous power is then calculated according to Equation 5.

$$
P(t)=\Sigma_{i=1}^{N_{\text {dampers }}} c_{i} V_{i}^{2}
$$

Coefficient $c_{i}$ uncertainty is defined by maximum and minimum coefficients, that are estimated by $C^{+}=\max \left(\frac{F_{V>0.02}}{V_{V>0.02}}\right)$ and $C^{-}=\min \left(\frac{F_{V>0.02 m / s}}{V_{V>0.02 m / s}}\right)$ (see Figure 4.b). A $0.02 \mathrm{~m} / \mathrm{s}$ threshold is defined in order to neglect data with high coefficients $(C \propto F / V)$ but very little dissipated power $(P \propto F . V)$.

The other method uses a segmented spline interpolation. The force signal is period-averaged, then filtered with a $5 \mathrm{~Hz}$ low-pass filter. Afterwards, it is divided into 12 segments according to the speed value $V$ and acceleration sign $\frac{\dot{V}}{|\dot{V}|}$. On each segment, a cubic spline interpolation is performed (Figure 4.a). The polynomial coefficients $k_{i, j}$ are registered to be used on the dissipated power estimation along with the trajectory data (Equation 6).

$$
P(t)=\Sigma_{i=1}^{N_{\text {dampers }}}([S][K][V]) . V_{i}
$$

In this equation, $[\mathrm{S}]$ is a row vector containing only 0 and a single 1 , used as a selector matrix indicating which coefficient should be used according to the speed value $V$ and acceleration sign $\frac{\dot{V}}{|V|}$. $[\mathrm{K}]$ is the $12 \times 4$ polynomial coefficients matrix and $[\mathrm{V}]$ is a column vector equal to $\left[1 ; V_{i} ; V_{i}^{2} ; V_{i}^{3}\right]$. 


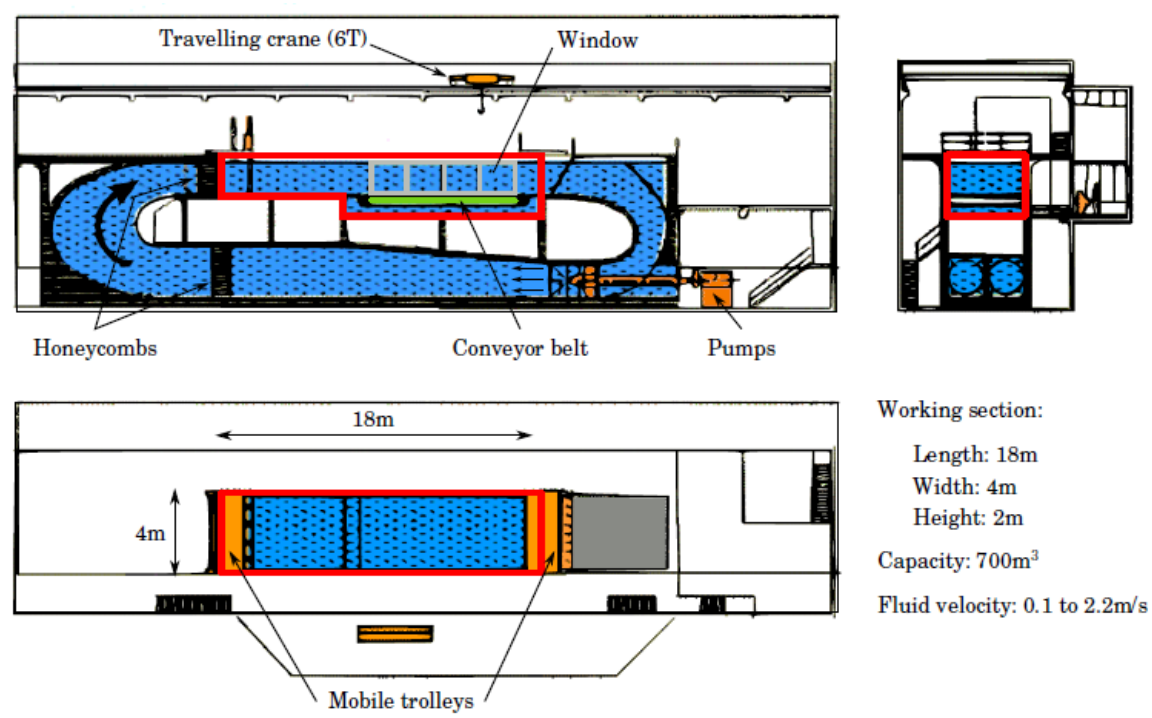

Figure 6: Drawing of Ifremer's flume tank of Boulogne-sur-Mer.

Experiments have been carried out in Ifremer flume tank of Boulognesur-Mer (Figure 6). Its working section is $18 \mathrm{~m}$ long, $4 \mathrm{~m}$ wide and $2 \mathrm{~m}$ deep. Flow velocity is adjustable up to $2.2 \mathrm{~m} / \mathrm{s}$. Here, trials have been made from $\mathrm{U}=0.7 \mathrm{~m} / \mathrm{s}$ up to $\mathrm{U}=1.2 \mathrm{~m} / \mathrm{s}$ with steps of $0.05 \mathrm{~m} / \mathrm{s}$ and with an upstream turbulence intensity rate of 3\% [Mycek et al. (2014)]. Fluid-Structure Interactions (FSI) simulations have been carried out to determine the size of the prototype according to the tank dimensions, so that the boundaries influence would be negligible. Blockage ratio is then $B=\frac{A_{\max } b}{S_{\text {tank }}}=0.08$. However, during trials one can observe circular surface perturbations behind the membrane at periodic interval, indicating an insufficient water height for wake characterisation without free surface effects $\left(H=2.5 A_{\max }\right)$. A window enables to see the membrane motion from the side, and to film it in order to record its trajectory. Six LEDs are located on the extremities of the dampers, and are used as targets for motion tracking. Therefore, a Qualysis Oqus 310 camera $\left(1024 \times 1296 \mathrm{px}^{2}\right)$ is placed at $1.5 \mathrm{~m}$ in front of the side window to film the trials at a frame rate of $f_{e}=40 \mathrm{~Hz}$ and a precision of $(\Delta x=10$ $\mathrm{mm}, \Delta y=10 \mathrm{~mm}$ ). Instrumentation also include a six-components load cell $\left(F_{z}^{\max }=18 \mathrm{kN}, F_{x, y}^{\max }=9 \mathrm{kN}\right)$ placed on the top of the frame (Figure 2.b) to measure forces and moments on the prototype. Sampling frequency for these transducers is $100 \mathrm{~Hz}$. Each trial lasts $\Delta t=80 \mathrm{~s}$, meaning that sampling size is $N=3200$ and frequency uncertainty is: $\Delta f=\frac{f_{e}}{N}=0.0125 \mathrm{~Hz}$. 


\section{Behaviour analysis}

In this section the dynamical behaviour of undulating membrane tidal energy converter is assessed. Trajectory, mode and frequency analysis are presented, then forces on the structure are displayed on a period. Finally, power estimations results are compared and evaluated. The reference configuration presented in this section is: $\mathrm{h}=3 \mathrm{~mm}, \mathrm{~d}=4,67 \%, \mathrm{C}_{2}=76660 \mathrm{~kg} / \mathrm{s}, \mathrm{U}=1$ $\mathrm{m} / \mathrm{s}$. Results are presented in non-dimensional form as: $U^{*}=U L^{2} \sqrt{\frac{\rho}{E \cdot b \cdot h^{3} / 12}}$; $x^{*}=\frac{x}{L} ; y^{*}=\frac{y}{L} ; t^{*}=t . f ; A^{*}=\frac{A}{L} ; f^{*}=\frac{f . L}{U} ; v_{p}^{*}=v_{p} / U ; F^{*}=\frac{F}{0.5 \cdot \rho_{f} \cdot L \cdot b \cdot U^{2}} ;$ $P^{*}=\frac{P}{0.5 \cdot \rho_{f} \cdot L \cdot b \cdot U^{3}}$.

a.

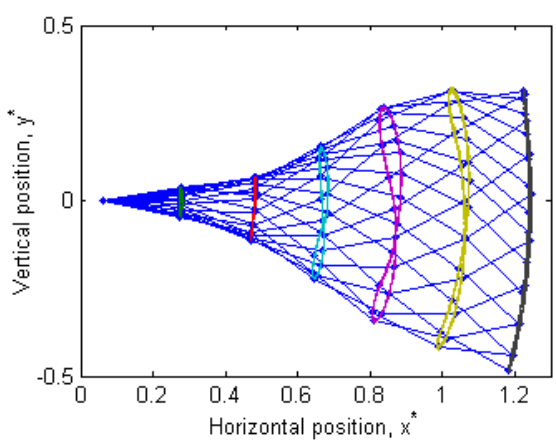

b.

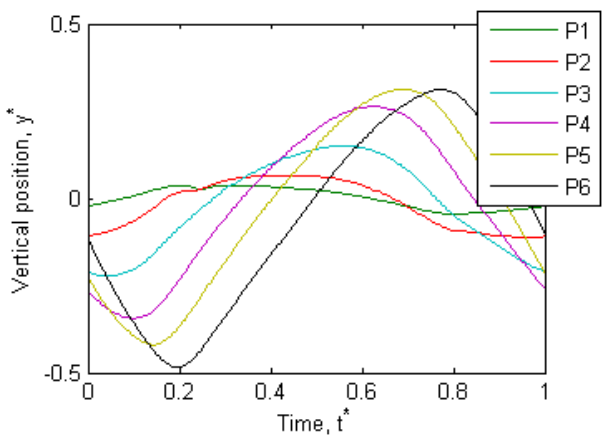

Figure 7: (a) Superimposed membrane's shapes measured each $1 / 20^{\text {th }}$ of a period.

(b) Vertical position of points on the membrane measured every $0.2 \mathrm{~L}$ during a period.

The results of motion tracking were obtained using the software Qualysis Track Manager, with automatic marker identification. Cubic interpolations were used for gaps smaller than 50 frames $(0.5 \mathrm{~s})$. Figure 7.a presents the trajectories of the six targets located along the membrane. Targets being fixed on the dampers' fixation $\left(x_{i}^{d}, y_{i}^{d}\right)$, the results in Figure 7 are extrapolated by : $x_{i}=x_{i}^{d}+h_{f i x} \sin \left(\alpha_{i}\right)$ and $y_{i}=y_{i}^{d}-h_{f i x} \cos \left(\alpha_{i}\right)$, with $\alpha_{i}=\arctan \left(\frac{y_{i+1}^{d}-y_{i-1}^{d}}{x_{i+1}^{d}-x_{i-1}^{d}}\right)$. The sixth point trajectory is fully an arc of a circle because its distance to the origin is limited by compression cables. The cables influence as well the other trajectories, that are a combination of an eight-shaped motion and an arc of a circle. For every points, the mean position is a little below $y^{*}=0$ due to the influence of gravity $\left(\rho>\rho_{f}\right)$. Figure 7.b shows the points' vertical position during a cycle. There is a propagating wave in the stream's direction with increasing amplitude. Vertical positions are close to sinusoids that rise slower than they go down, which is also due to gravity effect. Trajectory data enables to estimate the phase speed. This calculus gives $v_{p}^{*}=0.44^{ \pm 0.12}$. 
Wavelength $\lambda$ and wavenumber $k$ can also be estimated using the relation $\lambda=v_{p} / f=1.92^{ \pm 0.52} \mathrm{~m}$ and $k L=2 \pi L / \lambda=2.69^{ \pm . .57}$, indicating an undulation between mode $1(\mathrm{~kL}=1.876)$ and mode $2(\mathrm{~kL}=4.695)$ of a cantilever plate [Païdoussis (1998)].

a.

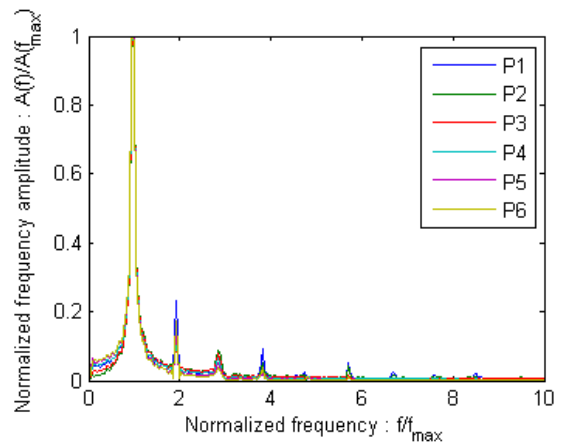

b.

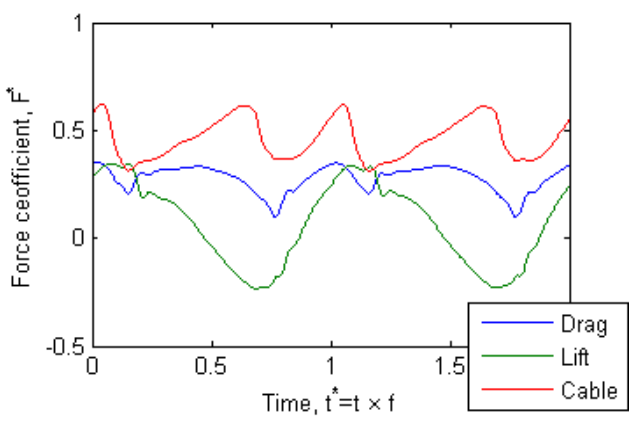

Figure 8: (a) Fourier transform of targets' vertical position at $U^{*}=8.5$. A zoom is presented in appendice A. (b) Force coefficients during two periods.

Figure 8.a shows the result of a discrete Fourier transform of the vertical position signal of each target. A zoom is available in appendix A. The transform results are normalized by the main frequency $\left(f_{\max }^{*}=0.184\right)$ and its amplitude, in order to compare the relative importance of harmonics. The main frequency is exactly the same for each target, enabling this comparison. Even harmonics are more important than odd ones. The harmonics -except for the first one- are relatively more important for upstream targets. This could be explained by an imperfect upstream clamping generating motion perturbations.

Forces on the membrane and in the cables are shown in Figure 8.b. Weight and forces on the frame were subtracted. Lift force signal is almost perfectly sinusoidal except for some perturbation at $t^{*} \approx 0.4$. It is not centred on zero, meaning that the structure is not vertically symmetric. Drag force signal has a doubled frequency and looks like a function of type: $F=\left|\sin \left(2 \pi t^{*}\right)\right|+K$. Cable forces also looks like an inclined sinusoid with a double frequency. Minimum cable force is close to: $F_{c}=\pi^{2} E I /(0.7 L)^{2}$, which is the critical buckling value for a clamped-pinned beam [Legay (2015)]. Cable tension is then the sum of the buckling force and the component of hydrodynamic force in the direction of the cables. 

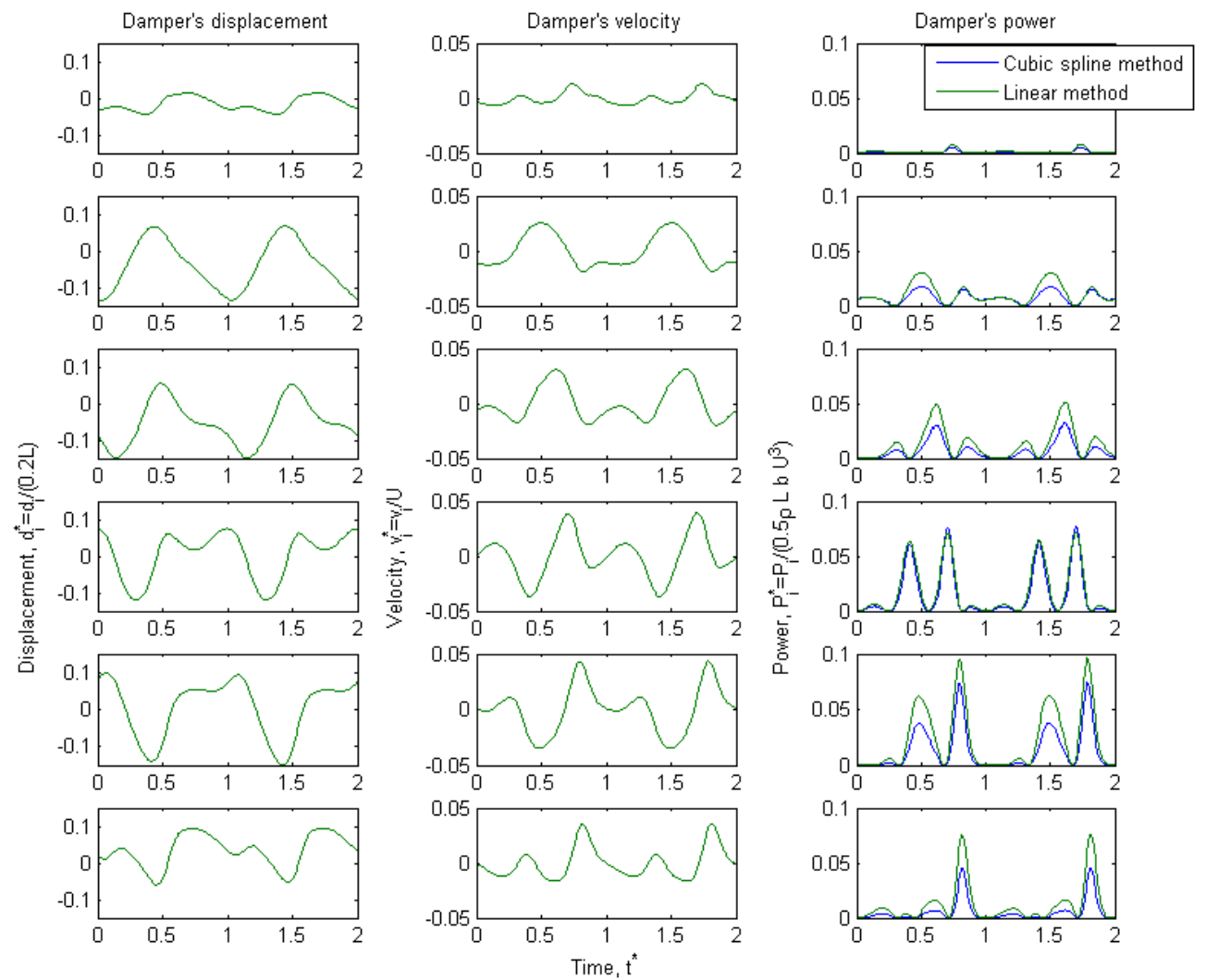

Figure 9: Dampers' displacement, velocity and power over two periods, ordered by damper's location (1 to 6$)$

The displacement of the dampers is measured directly, for the motion tracking targets are fixed on damper's fixations. The dampers' velocity is calculated with a simple centred finite difference scheme. Displacement and velocity signals, respectively in the left and middle column of Figure 9, are both filtered by a moving average with a $0.25 \mathrm{~s}$ window. The velocity range measured during the trial $( \pm 0.05 \mathrm{~m} / \mathrm{s})$ matches the velocity range used in the dampers calibration. The column on the right of Figure 9 represents the power dissipated on each segment during two periods, according to both methods. It is then the sum of the power dissipated by the four dampers placed on the same position along the membrane. The cubic spline interpolation tends to underestimate overall power production in comparison to the linear method. 
Total mean power coefficient is calculated over ten periods. It is estimated to be $P_{\text {spline }}^{*}=0.057$ by the cubic-spline method (Equation 6) and $P_{\text {linear }}^{*}=0.0817^{ \pm 0.069}$ by the linear method (Equation 5). Power production is concentrated in some instants of the cycle (Figure 10.a) and the majority of contribution is made by segments $n^{\circ} 4$ and $n^{\circ} 5$ as confirmed by Figure 10.b. Operational optimization should then oversee that the power is produced more uniformly along the membrane and over the whole cycle.

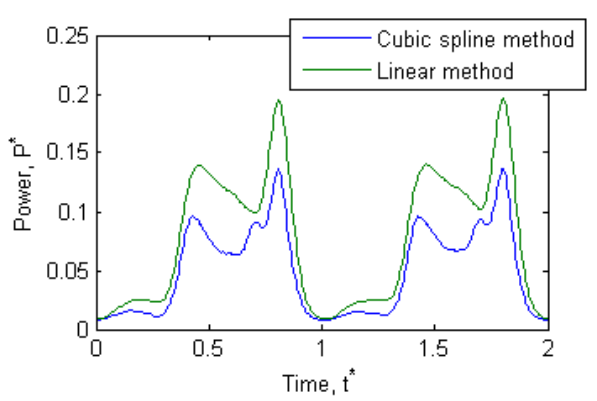

b.

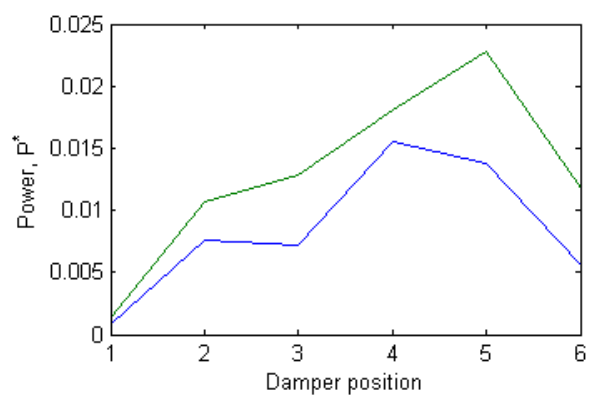

Figure 10: (a) Power dissipation over two periods. (b) Damper contribution calculated over ten periods by both methods used for power estimation

In order to evaluate the power estimation methods, a damper was randomly selected and tested on the displacement table with the six trajectories measured for the upper dampers. The damper here is adjusted at $c=2906$ $\mathrm{kg} / \mathrm{s}$. Forces recorded on the damper as well as those computed by linear and segmented cubic spline methods are presented on Figure 11. Both estimations approximate quite well the real damping force. However, they tend to overestimate damping force for negative velocity and are less precise during force peaks and around $F_{i}^{*}=0$. The spline method also presents some shifts for low forces. It results from sudden coefficients transitions (see Eq. 6).

Power is obtained by calculating the mean value of the product of force estimation (or measurement) and velocity signal during two periods. Mean relative errors on power estimations are then: $\xi_{\text {lin }}=\Sigma_{i=1}^{N=6}\left(\frac{P_{\text {lin }}-P_{\text {real }}}{N . P_{\text {real }}}\right)=-0.17$ and $\xi_{\text {spline }}=\Sigma_{i=1}^{N=6}\left(\frac{P_{\text {spline }}-P_{\text {real }}}{N . P_{\text {real }}}\right)=-0.24$. 
The behaviour of the selected damper is representative of all the others and the errors calculated here correspond to an average operating point, so it can be considered as an insight of the power calculation precision. Linear method seems to be more precise than the cubic spline one but both will be used in the next part. Indeed, the spline power estimation takes into account phenomena like hysteresis, quadratic behaviour and asymmetry. It is also more conservative on some force peaks. Using both methods enables to compare the tendencies of the results and to give a better insight of the high uncertainty.
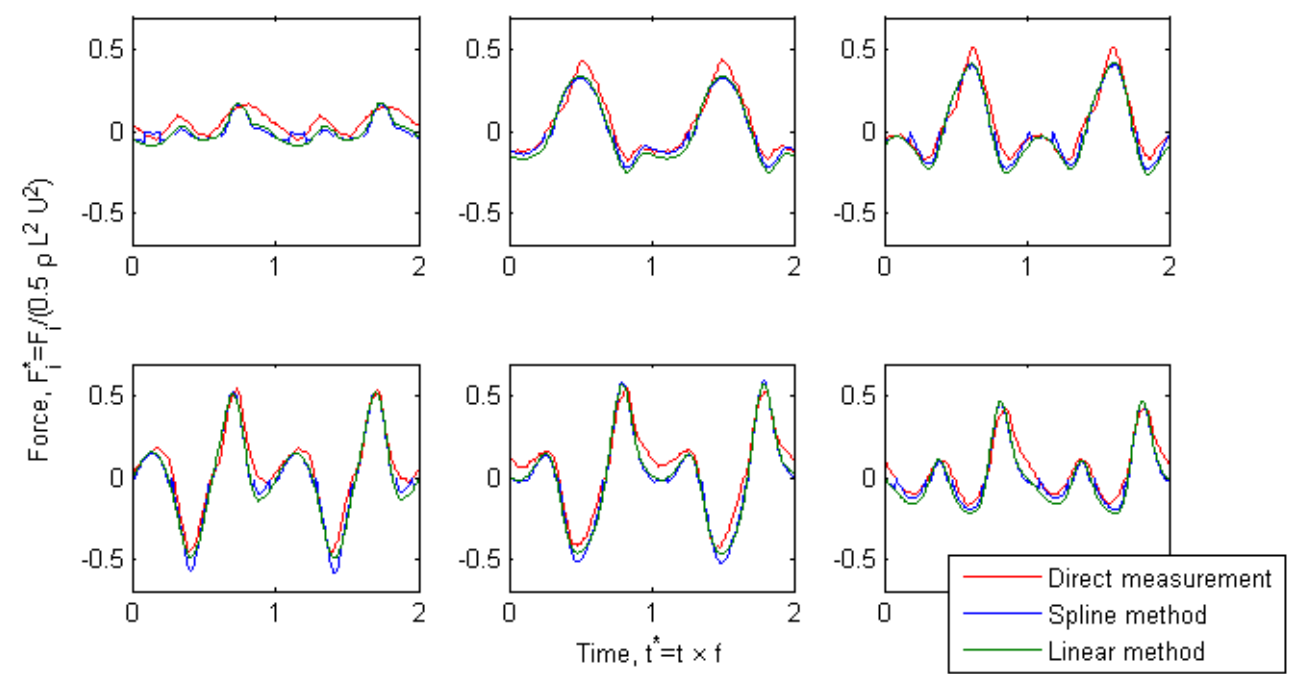

Figure 11: Force estimation verification with the six measured upper trajectories for a randomly selected damper

A way to lower this uncertainty would be to use dampers with a more linear behaviour or to test all of them on the displacement table with all the trajectories that have been measured during the membrane tank tests. This last solution would be too time-consuming, therefore the error order of magnitude is considered acceptable in the next section. 


\section{PTO and current speed variation}

The behaviour of the membrane has been caracterised and presented for one specific configuration. In this section, the results of trajectory, forces and power estimation will be presented for different damping rates and reduced current speed. Damping parameters $C$ are given in Table 1 and reduced speed is calculated according to Eq. 7. It is a non dimensional parameter frequently used in fluid-structure interactions literature and is proportional to the ratio between flow speed and the celerity of elastic waves in the structure. Current speed varies from critical speed $U_{c}^{*}$, that is the cut-in speed from which the membrane starts undulating, up to $U^{*} \approx 7$. Results are averaged over as many periods there is in their sample, with Eq. 8. The minimum sample size is $N_{\text {period }}=7$. Standard deviations are plotted as error bars (Eq. 9, with $X_{i}$ being the value $X$ calculated on during $i$-th period and $\bar{X}$ the average over $N_{\text {period }}$ periods).

$$
\begin{gathered}
U^{*}=U L^{2} \sqrt{\frac{\rho}{E . b \cdot h^{3} / 12}} \\
N_{\text {period }}=\lfloor f . \Delta t\rfloor \\
\sigma(X)=\sqrt{\frac{1}{N_{\text {period }}} \sum_{i=1}^{N_{\text {period }}}\left(X_{i}-\bar{X}\right)^{2}}
\end{gathered}
$$
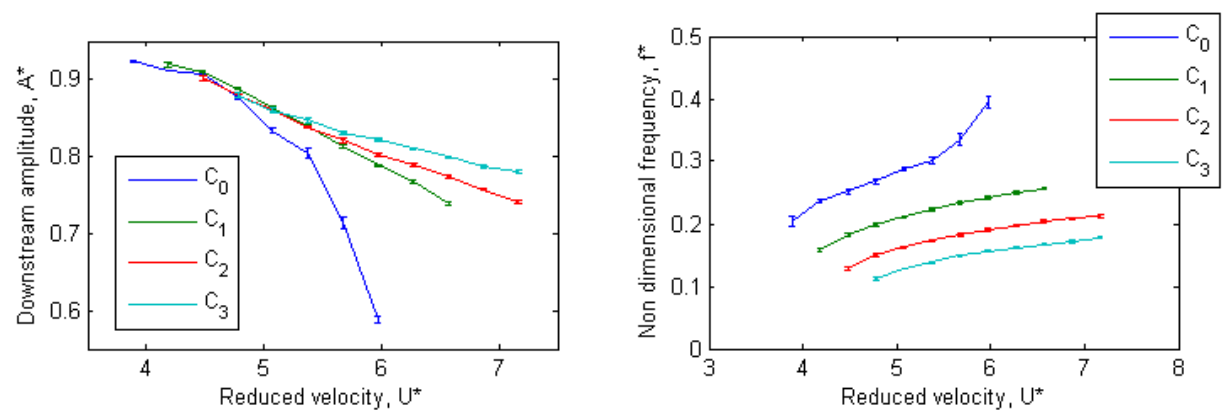

Figure 12: (a) Maximal amplitude and (b) main frequency results for different damping coefficients and fluid velocity 
Figure 12 displays in the left the results of maximum amplitude and in the right the main frequency. In all tested configurations, maximum target amplitude is the downstream one. Firstly, it shows the critical speed rising according to damping rate [Träsch et al. (2016)]. Secondly, the amplitude tends to decrease when current speed is higher and to increase when there is more dampers, on the opposite to main frequency. This opposite behaviour suggests that there is a balance to find between frequency and amplitude, adjusting damping according to fluid velocity.

Also, the trajectory is similar to what was presented in Figure 7.a in all case except $C_{0}$ at $U^{*}>6.5$. Indeed, the undamped membrane $\left(C_{0}\right.$, blue line) has a behaviour different from configurations with dampers. It shows the same tendencies in maximal amplitude and main frequency, but with higher variations above $U^{*}=6.5$. It is a sign of a mode change above this velocity. Indeed, trajectory analysis reveals here a node around target $\mathrm{n}^{\circ} 3$, while it is elsewhere similar to what was presented in Figure 7.

a.

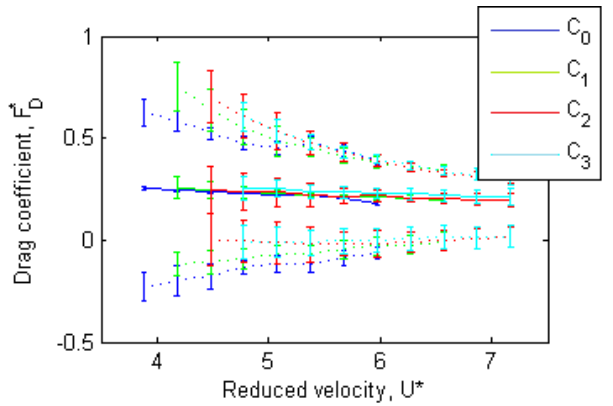

b.

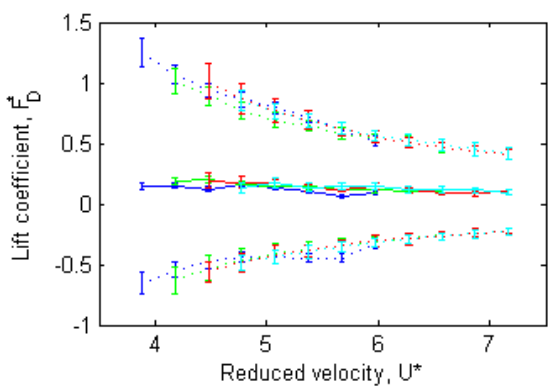

Figure 13: (a) Drag and (b) lift forces on the membrane. Mean values: full line, maximal and minimal values: dotted line

Forces and power coefficients are calculated according to the membrane's area $L \times b$ (Eq. $10 \& 11)$. Their meaning differs from what is commonly used in tidal energy, where the power coefficient is the ratio between mechanical power and incident hydrokinetic power. However, this criteria enables to use a fixed surface for coefficient calculation (on contrary to the swept area, $b \times A$, which depends on other parameters such as flow speed).

$$
F^{*}=\frac{F}{0.5 \cdot \rho_{f} \cdot L \cdot b \cdot U^{2}}
$$




$$
P^{*}=\frac{P}{0.5 \cdot \rho_{f} \cdot L \cdot b \cdot U^{3}}
$$

Drag and lift coefficients are presented in Figure 13. These forces are usefull for the design of the future large scale device's support. The signals being periodic, only mean and extreme values are plotted. In both graphics, we can notice that the amplitude of these forces is much more important than signals' averages. Mean drag coefficient is a little higher for high damping, whereas it decreases when water current is faster. Drag amplitude is also significantly lower for fast currents, we noticed that $\max \left(F_{D}\right)$ evolves proportionately to $U$, while $\overline{F_{D}^{*}}$ is proportional to $U^{2}$.

Lift is the dominant force in the system, as illustrated by Figure 13.b. Lift coefficient amplitude is higher than 1 for small fluid velocity, then strongly decrease (as $\max \left(F_{L}\right)$ is also proportional to $U$ ). Here as well, mean lift coefficient is positive, as explained for Figure 8. Explication could also include an undesired influence of the frame's wake on the membrane or the influence of the free surface and/or the tank's bottom.

Results of power coefficient are plotted in Figure 14.a. The linear method results are presented in dashed line, whereas the spline method results are presented in full line. The linear method power coefficient is greater of around $30 \%$, but both methods do give the same tendencies. There is a little increase in power according to damping and no significant variation depending on fluid velocity between $U^{*}=U_{c}^{*}$ and $U^{*}=6$. Above this value, power conversion starts to decrease. For this configuration, best performance should be with damping greater than $C_{3}$ and for relatively low flow speed (around $U^{*} \approx 5$ ).

a.
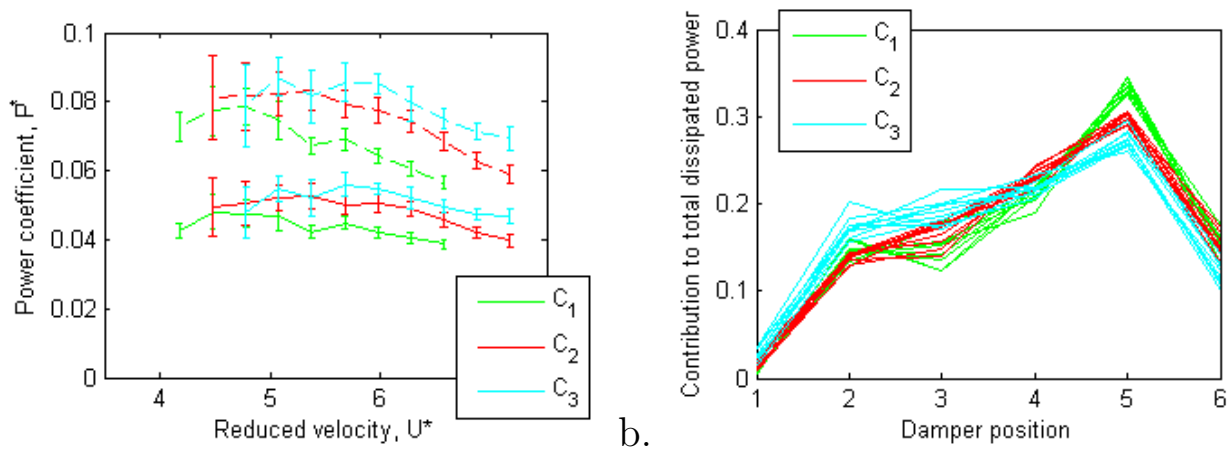

Figure 14: (a) Power coefficient in relation to damping and fluid velocity for both methods. Dashed line: $P_{\text {linear }}^{*}$, full line: $P_{\text {spline }}^{*}(\mathrm{~b})$ Dampers contribution to total dissipated power for every test configuration. 
Power coefficient is quite steady according to reduced flow speed at low flow speed $\left(U^{*}<6\right)$. On the other hand, it decreases a little for higher values of $U^{*}$. Power coefficient is normalized by $U^{3}$. Other parameters in its calculation are steady during all experiments, as well as those used for reduced flow speed (Equations 7 and 11). Results then suggest that estimated converted power scales according to $U^{3}$ at low flow speed.

Figure 14.b displays the contribution to total dissipated power according to dampers' position on the membrane. The majority of power is still harvested by dampers $n^{\circ} 4$ and $n^{\circ} 5$, while the first damper has almost no production. It can be explained by the membrane's limit conditions: upstream clamping limits the amplitude of the upstream part of the membrane while the free end restricts deformation on the membrane's downstream part. Figure 14.b also demonstrates that increasing damping leads to a more uniform power production with a higher participation of damper $n^{\circ} 2$ and $n^{\circ} 3$ and a lower of $n^{\circ} 5$. It is also possible to increase power dissipation by adjusting other parameters: membrane's Young modulus $E$, cable length $d$, hanging conditions or damping repartition [Träsch et al. (2016)].

It was earlier demonstrated that damping increases undulation amplitude and lowers its main frequency. Yet, these two parameters take part in power dissipation. First one impacts dampers strokes, the other one their working speed. Thus, simplifying dampers motion to a sinusoid and assuming that their stroke is proportional to the membrane's global amplitude, there is : $V_{i} \propto 2 \pi f . A \cdot \sin (2 \pi f)$. Moreover, $P=\Sigma F_{i} \cdot V_{i}=\Sigma C_{i} \cdot V_{i}^{2}$. To verify this link, $P /\left(C .(f . A)^{2}\right)$ is plotted in function of the reduced velocity in Figure 15 . This graphic shows indeed that for the estimated power, at fixed damping, this parameter is almost constant, or that $P \propto(f . A)^{2}$. This relation could be used for fast power estimation, but needs further investigation beforehand.

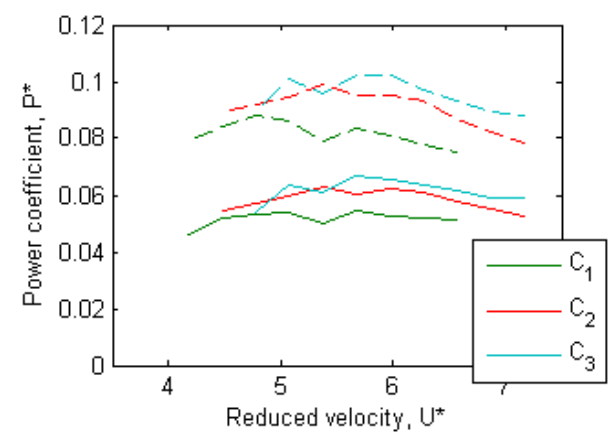

Figure 15: Relation between dissipated power and C.(f.A $)^{2}$. Dashed line: $P_{\text {linear }}^{*}$, full line: $P_{\text {spline }}^{*}$ 


\section{Conclusion}

An innovative concept of tidal energy device has been presented. It uses undulation occurring from interaction between a semi-rigid plate and an uniform flow. The concept of this device has been proven by designing a $1 / 20^{t h}$ prototype and testing it in a flume tank. The prototype is made of a polyacetal membrane reinforced by transverse composite bars and prestressed by lateral cables. Hydraulic dampers are used to model power conversion. Instrumentation include trajectory record and force measurement. The dampers have been characterized on a displacement table. They have a non-linear behaviour including hysteresis, non-symmetry, and quadratic component. A representative damping coefficient has been introduced to describe their impact, which is an increase in amplitude and a frequency decrease, in opposition to fluid velocity's influence.

The prototype behaviour have been analysed for a reference configuration. A propagating wave has been observed in the flow direction. Undulation mode, frequency and forces on the structure have been described. Two power estimation methods have been presented and evaluated. The linear method, assuming pure damping of the hydraulic dampers, seems to be more precise. The other one is based on a segmented cubic spline interpretation and attempt to take into account the dampers particularities (asymmetry, quadratic behaviour, hysteresis). It results in lower standard deviation but also generates important errors that can be due to non-representative calibration. The device has then been tested for different damping rates and flow speed. Motion amplitude, frequency, forces and power estimation results have been presented. Power repartition on the membrane shows a higher contribution from the downstream half of the membrane. Power estimation results shows that it scales with the cube of flow speed and with the square of the frequency-amplitude product.

Further investigations will focus on the improvement of the PTO system, with the specific development of linear dampers at the right scale. A parametric study is also ongoing on the impact of membrane's stiffness, hanging condition, cable length and damping repartition. Once the small scale prototype's behaviour will be closer to the full scale device, a wake characterisation and an interaction study between several prototypes will be undertaken. 


\section{Acknowledgement}

The authors gratefully acknowledge the French Environment and Energy Management Agency (ADEME) and EEL Energy for their financial support. They also want to express their full gratitude to J.-V. Facq and T. Bacchetti for their assistance and advice during trials.

Appendix A. Fourier transform of target's position at $U^{*}=8.5$. A zoom of Figure 8.a.

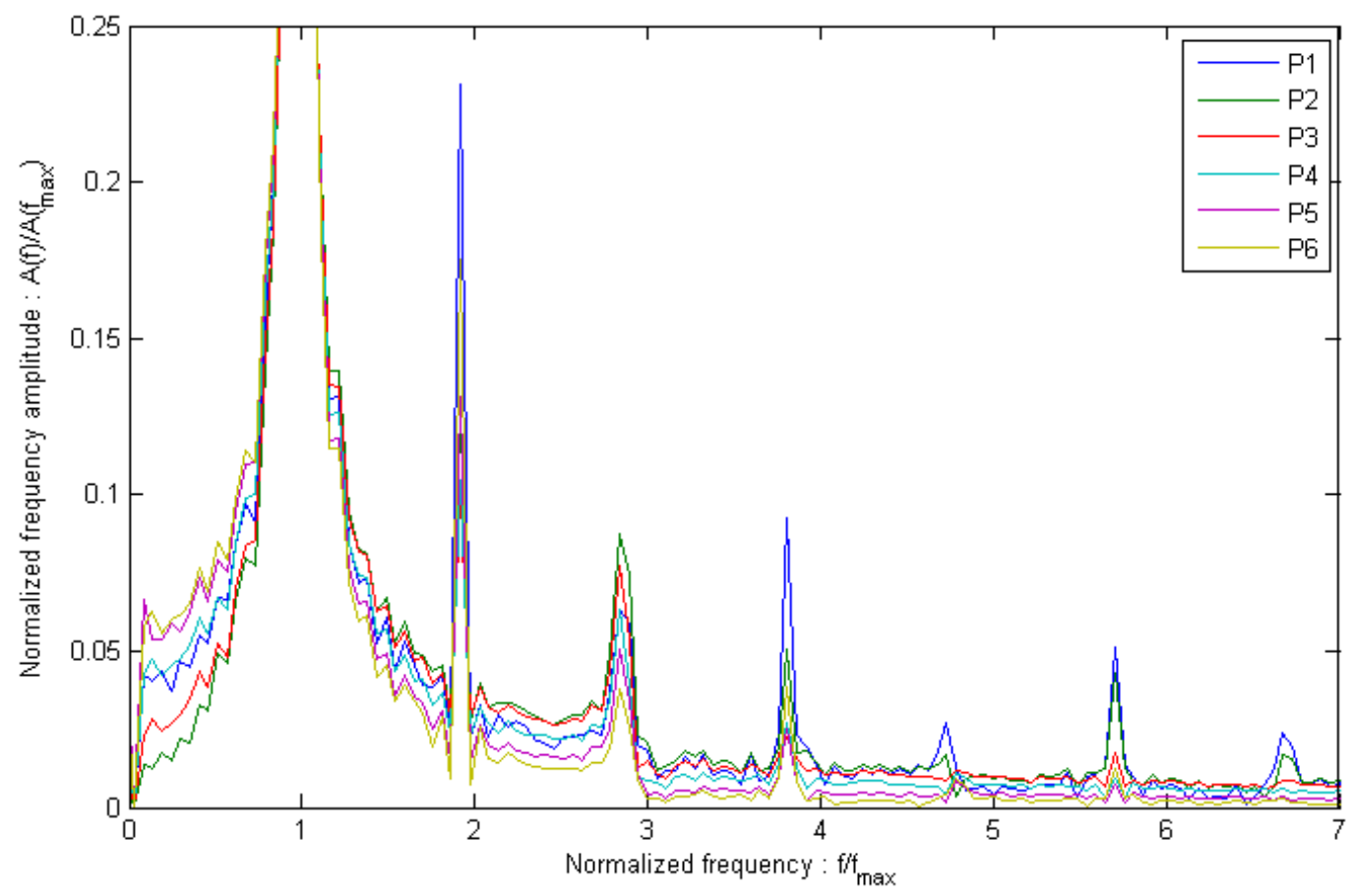




\section{Nomenclature}

\begin{tabular}{|c|c|c|}
\hline$A$ & Maximum amplitude & $m$ \\
\hline$b$ & Membrane width & $m$ \\
\hline$B$ & Blockage ratio & $\%$ \\
\hline$c$ & Damping coefficient (damper) & $\mathrm{kg} / \mathrm{s}$ \\
\hline$C$ & Damping coefficient (membrane) & $\mathrm{kg} / \mathrm{s}$ \\
\hline$d$ & Cable withdraw length & $m$ \\
\hline E & Young's modulus & $P a$ \\
\hline$f_{e}$ & Sampling rate & $H z$ \\
\hline$f$ & Frequency & $H z$ \\
\hline$F$ & Force & $N$ \\
\hline$F_{c}$ & Euler critical buckling force & $N$ \\
\hline$F_{d}$ & Drag force & $N$ \\
\hline$F_{l}$ & Lift force & $N$ \\
\hline$h$ & Membrane thickness & $m$ \\
\hline$h_{f i x}$ & Damper's fixation height & $m$ \\
\hline$H$ & Tank height & $m$ \\
\hline$I$ & Second moment of inertia & $m^{4}$ \\
\hline$k$ & Wavenumber & $m^{-1}$ \\
\hline$k_{\text {arm }}$ & Arms' stiffness & $N / m$ \\
\hline$K$ & Constant & . \\
\hline$L$ & Membrane lengt & $m$ \\
\hline$L_{\text {flap }}$ & Flap length & $m$ \\
\hline$N$ & Sample size & . \\
\hline$N_{\text {dampers }}$ & Dampers number & \\
\hline$P$ & Power & $W$ \\
\hline$P_{\text {int }}$ & Intern pressure & $P a$ \\
\hline$P_{\text {vap }}$ & Vapour pressure & $P a$ \\
\hline$\Delta P$ & Pressure drop & $\mathrm{Pa}$ \\
\hline$S_{\text {tank }}$ & Tank cross-section & $m^{2}$ \\
\hline$t$ & Time & $s$ \\
\hline$T$ & Period & $s$ \\
\hline$U_{c}$ & Critical speed (or cut-in speed) & $\mathrm{m} / \mathrm{s}$ \\
\hline$U$ & Current speed & $\mathrm{m} / \mathrm{s}$ \\
\hline$v_{p}$ & Phase speed & $\mathrm{m} / \mathrm{s}$ \\
\hline$V$ & Velocity & $\mathrm{m} / \mathrm{s}$ \\
\hline$x$ & Axial coordinate & $m$ \\
\hline$y$ & Vertical coordinate & $m$ \\
\hline$z$ & Damper's displacement & $m$ \\
\hline$\alpha$ & Local angle to horizon & $\mathrm{rad}$ \\
\hline$\lambda$ & Wavelength & $m$ \\
\hline$\rho$ & Membrane density & $\mathrm{kg} / \mathrm{n}$ \\
\hline$\rho_{f}$ & Fluid density & $\mathrm{kg} / \mathrm{r}$ \\
\hline$\sigma$ & Standard deviation & $\%$ \\
\hline
\end{tabular}




\section{References}

A.H. Day, A. Babarit, A. Fontaine, Y-P. He, M. Kraskowski, M. Murai, I. Penesis, F. Salvatore, H-K. Shin. Hydrodynamic modelling of marine renewable energy devices: A state of the art review. Ocean Engineering, 2015.

A. Déporte. Caractérisation du fonctionnement d'une hydrolienne à membrane ondulante pour la récupération de l'énergie des courants marins PhD. Thesis, Université de Bretagne Occidentale, Dec. 2015.

O. Doare and S. Michelin. Piezoelectric coupling in energy-harvesting fluttering flexible plates: linear stability analysis and conversion efficiency. Journal of Fluids and Structures, Nov. 2011.

A. Jbaily, R. W. Yeung. Piezoelectric devices for ocean energy: a brief survey Journal of Ocean Engineering and Marine Energy, Volume 1, Issue 1, pp 101-118, Feb. 2015.

T. Kinsey, G. Dumas, G. Lalande, J. Ruel, A. Mehut, P. Viarouge, J. Lemay, and Y. Jean. Prototype testing of a hydrokinetic turbine based on oscillating hydrofoils. Renewable Energy, 36(6):1710-1718, Jun. 2011.

A. Legay Mechanics of materials. Lecture notes, CNAM Paris, 2015-2016.

P. Mycek, B. Gaurier, G. Germain, G. Pinon, E. Rivoalen. Experimental study of the turbulence intensity effects on marine current turbines behaviour. Part I: One single turbine. Renewable Energy, Vol. 66, 729-746, Jun. 2014.

M. P. Païdoussis. Fluid-Structure Interactions vol. 1: Slender structures and axial flow. Academic Press, 1998.

G. Taylor, J. Burns, S. Kammann, W. Powers, T. Welsh. The Energy Harvesting Eel: A Small Subsurface Ocean/River Power Generator. IEEE Journal of Oceanic Engineering, Vol. 26, N. 4, Oct. 2001.

M. Träsch, A. Déporte, G. Germain, B. Gaurier, J-B. Drevet. Impact of structural stiffness on undulating energy tidal energy converter dynamics. Journées de l'Hydrodynamique, Brest, 2016.

M. Träsch, A. Déporte, G. Germain, J-B. Drevet. Impact of cables withdraw length on the dynamics of an undulating membrane tidal energy converter. EWTEC, 2017.

J. Verne. Vingt mille lieues sous les mers. J. Hetzel.

P.-L. Viollet. Histoire de l'énergie hydraulique. Paris : Presses de l'École nationale des ponts et chaussées, DL 2005

Q. Xiao and Q. Zhu. A review on flow energy harvesters based on flapping foils. Journal of Fluids and Structures, 46:174-191, Apr. 2014. 